\title{
Comparison of the Efficacy of Piperacillin/Tazobactam and Meropenem, with or without Intravenous Immunoglobulin, as Second-Line Therapy for Febrile Neutropenia: A Prospective, Randomized Study
}

\author{
Hirozumi Sano, Ryoji Kobayashi, Satoru Matsushima, Daiki Hori, \\ Masato Yanagi, Koya Kodama, Daisuke Suzuki and Kunihiko Kobayashi \\ Department of Hematology/Oncology for Children and Adolescents, Sapporo Hokuyu Hospital, Sapporo, Japan
}

Background: Febrile neutropenia (FN) remains an important complication in pediatric cancer patients. The present study compared the efficacy of meropenem (MEPM) and piperacillin/tazobactam (PIPC/TAZ) with or without intravenous immunoglobulin (IVIG) as second-line therapy for FN in pediatric patients.

Methods: As first-line treatment for FN, 394 episodes in 99 patients were randomly assigned to receive PIPC/TAZ $(360 \mathrm{mg} / \mathrm{kg} /$ day, maximum $18 \mathrm{~g} /$ day) or MEPM (120 mg/ $\mathrm{kg} /$ day, maximum $3 \mathrm{~g} /$ day). Eighty-four episodes in 42 patients were judged as failures, and, thus, were enrolled for second-line treatment. In second-line treatment, antibiotics were switched to MEPM or PIPC/TAZ, and episodes were further randomized for treatment with or without concomitant IVIG at $100 \mathrm{mg} / \mathrm{kg} / \mathrm{day}$ (maximum $5 \mathrm{~g} /$ day) for 3 consecutive days.

Results: The total success rate of second-line treatment was $50.0 \%$ (52.0\% in PIPC/ TAZ and $47.2 \%$ in MEPM with or without IVIG, $P=0.826$ ). The success rates of patients treated with (IVIG+ group) and without IVIG (IVIG - group) were 53.8 and $46.7 \%$, respectively $(P=0.662)$. In the IVIG + group, the success rate of patients younger than 8 years old was $78.6 \%$, which was significantly higher than that of those aged 8 years and older (40.0\%, $P=0.043)$.

Conclusion: PIPC/TAZ and MEPM were equally effective as second-line treatment. Concomitant IVIG was also effective, particularly in patients younger than 8 years.

Key Words: Meropenem, Piperacillin/Tazobactam, Immunoglobulin, Febrile neutropenia, Second-line treatment
pISSN 2233-5250 / elSSN 2233-4580 https://doi.org/10.15264/cpho.2021.28.2.75 Clin Pediatr Hematol Oncol 2021;28:75 83

Received on May 31, 2021 Revised on July 27, 2021 Accepted on September 10, 2021
Corresponding Author: Hirozumi Sano
Department of Hematology/
Oncology for Children and
Adolescents, Sapporo Hokuyu
Hospital, Higashi-Sapporo 6-6,
Shiroishi-ku, Sapporo 003-0006,
Japan
Tel: +81-11-865-0111
Fax: +81-11-865-9719
E-mail: hirozumi.sano@gmail.com
ORCID ID: orcid.org/0000-0003-4183-4602

\section{Introduction}

The clinical outcomes of pediatric patients with hematological and malignant diseases have improved in recent years; however, life-threatening bacterial infections occur in approximately $5-10 \%$ of these patients during episodes of febrile neutropenia (FN) [1,2]. Several guidelines recommend piperacillin/tazobactam (PIPC/TAZ), cefepime, and carbapenems as first-line antibiotic therapy for adult patients at a high risk of FN. However, limited information is currently available on second-line antibiotic therapy for FN. We previously conducted two prospective studies on second-line antibiotic therapy for FN [3,4]. The first study showed the usefulness of meropenem (MEPM) as second-line antibiotic therapy as well as concomitant intravenous immunoglobulin (IVIG) [3]. The second study revealed that PIPC/TAZ was as effec- 
tive and safe as MEPM for second-line therapy for FN, and also demonstrated the efficacy of IVIG, particularly for patients with low serum immunoglobulin G (IgG) levels [4]. The present prospective, randomized study was performed to compare the efficacies of MEPM and PIPC/TAZ with or without concomitant IVIG, which were used as second-line treatment for pediatric patients with FN. The comparison of MEPM and PIPC/TAZ performed in the present study was the same as that in the previous study [4]; however, the dose and administration frequency of PIPC/TAZ changed from the previous study in first- and second-line treatments [5]. The objective of the present study was to investigate changes in patient outcomes by increasing the doses of and changing in the administration procedure of PIPC/TAZ in second-line antibiotic treatment.

\section{Materials and Methods}

\section{1) Patient eligibility and study design}

The present study included febrile and neutropenic patients who underwent conventional or high-dose chemotherapy including hematopoietic stem cell transplantation (HSCT) for hematological malignancies or solid tumors at the Department of Hematology/Oncology for Children and Adolescents, Sapporo Hokuyu Hospital between April 2016 and March 2020. Regarding first-line treatment, $394 \mathrm{FN}$ episodes in 99 patients were randomly assigned to receive PIPC/TAZ $(360 \mathrm{mg} / \mathrm{kg} /$ day in 4 doses, 1-hour drip infusion, maximum $18 \mathrm{~g} /$ day) or MEPM $(120 \mathrm{mg} / \mathrm{kg} /$ day in 3 doses, 1 -hour drip infusion, maximum $3 \mathrm{~g} /$ day). The randomization of the present study was performed in accordance with CONSORT 2010 guideline [6] (CONSORT 2010 checklist of information to include when reporting a randomized trial was shown in Supplementary Table 1, CONSORT 2010 Flow Diagram was shown in Supplementary Table 2A (patients treated with MEPM) and 2B (patients treated with PIPC/TAZ)). Simple randomization (1:1) with stratification was performed. Risk stratification by age, gender, underlying disease status, the duration of neutropenia, severity of neutropenia, or any other factor was not conducted. Alloca- tions were performed by medical staff. Randomization was performed in an evaluator-blind manner, and the treatment allocation remained unknown to the investigators who assessed outcomes and adverse events throughout the present study. In 24 out of 393 FN episodes, blood cultures before the initiation of first-line treatment were positive. Patients were eligible if they met the following criteria: (1) fever defined as a temperature of higher than $37.5^{\circ} \mathrm{C}$ for at least 1 hour or a single temperature higher than $38^{\circ} \mathrm{C}$; (2) an absolute neutrophil count (ANC) of less than $0.5 \times 10^{9} / \mathrm{L}$; and (3) no preceding administration of antibiotics within 72 hours prior to the initiation of treatment, except for trimethoprim-sulfamethoxazole as prophylaxis for Pneumocystis jirovecii infection. Clinical efficacy was evaluated at 120 hours (in accordance with the 2002 Guidelines of the Infection Disease Society of America, we selected 120 hours as the time for an evaluation following the initiation of antibiotic therapy [7]). Treatment outcome criteria were defined as follows. Success was defined as the disappearance of fever, clinical improvement, eradication of the infecting organism, and maintenance of a response for at least 7 days after the discontinuation of the antibiotic treatment. Failure was defined as the persistence of fever or infecting organisms, a required change in the antibiotic treatment, new infection, or deterioration/death due to infection. In first-line treatment, 105 episodes were judged as failures (PIPC/TAZ: 44 episodes, MEPM 61 episodes). Among these episodes, 19 did not proceed to second-line treatment because of death in 1 episode and a change in antibiotics in the remaining 18. Eighty-six episodes in 44 pediatric patients were enrolled for second-line treatment. Patients were randomly assigned to receive PIPC/TAZ or MEPM with or without IVIG at $100 \mathrm{mg} / \mathrm{kg} /$ day (maximum dose: $5 \mathrm{~g} /$ day) for 3 consecutive days (Venoglobulin IH 5\% I.V., Japan Blood Products Organization, Tokyo, Japan). MEPM was administered at $120 \mathrm{mg} / \mathrm{kg} /$ day (maximum dose: $3 \mathrm{~g} /$ day) in a 1-hour drip infusion 3 times a day for patients who received PIPC/TAZ as first-line treatment. PIPC/TAZ was administered at $360 \mathrm{mg} / \mathrm{kg} /$ day (maximum dose: $18 \mathrm{~g} /$ day) in a 1-hour drip infusion 4 times a day for patients 
who received MEPM as first-line treatment (Fig. 1).

Evaluations before the initiation of each antibiotic treatment included sex, age, body weight, type of underlying disease, and history of preceding HSCT within 3 months of the FN episode. Laboratory examinations consisting of a complete blood count, peripheral blood smear, quantitative $C$-reactive protein, $\beta-D$ glucan, procalcitonin, liver and renal functions, urinalysis, and blood cultures from specimens obtained via a peripheral venous puncture and/or central venous (CV) access device, if in place, were conducted. Routine chest and abdominal computed tomography (CT) scans were also performed before the initiation of second-line treatment. If invasive fungal infection (IFI) was suspected based on laboratory or CT findings, antifungal therapy (micafungin or liposomal amphotericin) was used in addition to second-line treatment. This treatment was continued until the completion of an appropriate course of therapy for a defined clinical or microbiological infection. The efficacy of second-line treatment was evaluated 72 hours after its initiation (Fig. 1).

The present study was performed in compliance with the updated Declaration of Helsinki. The Institutional Review Board of Sapporo Hokuyu Hospital approved this study, and written informed consent was obtained from all patients or their parents.

\section{2) Definitions and infection prophylaxis}

Fever was defined as an axillary temperature of $37.5^{\circ} \mathrm{C}$ or higher on two occasions at least 1 hour apart or a single axillary temperature higher than $38.0^{\circ} \mathrm{C}$. Neutropenia was defined as ANC $<0.5 \times 10^{9} / \mathrm{L}$, and severe neutropenia was also defined in the present study as ANC $<0.1 \times$ $10 \%$ L. Bacteremia was defined as a state in which a single bacterial organism was isolated from the blood of a patient. When bacteria that typically colonize the skin,

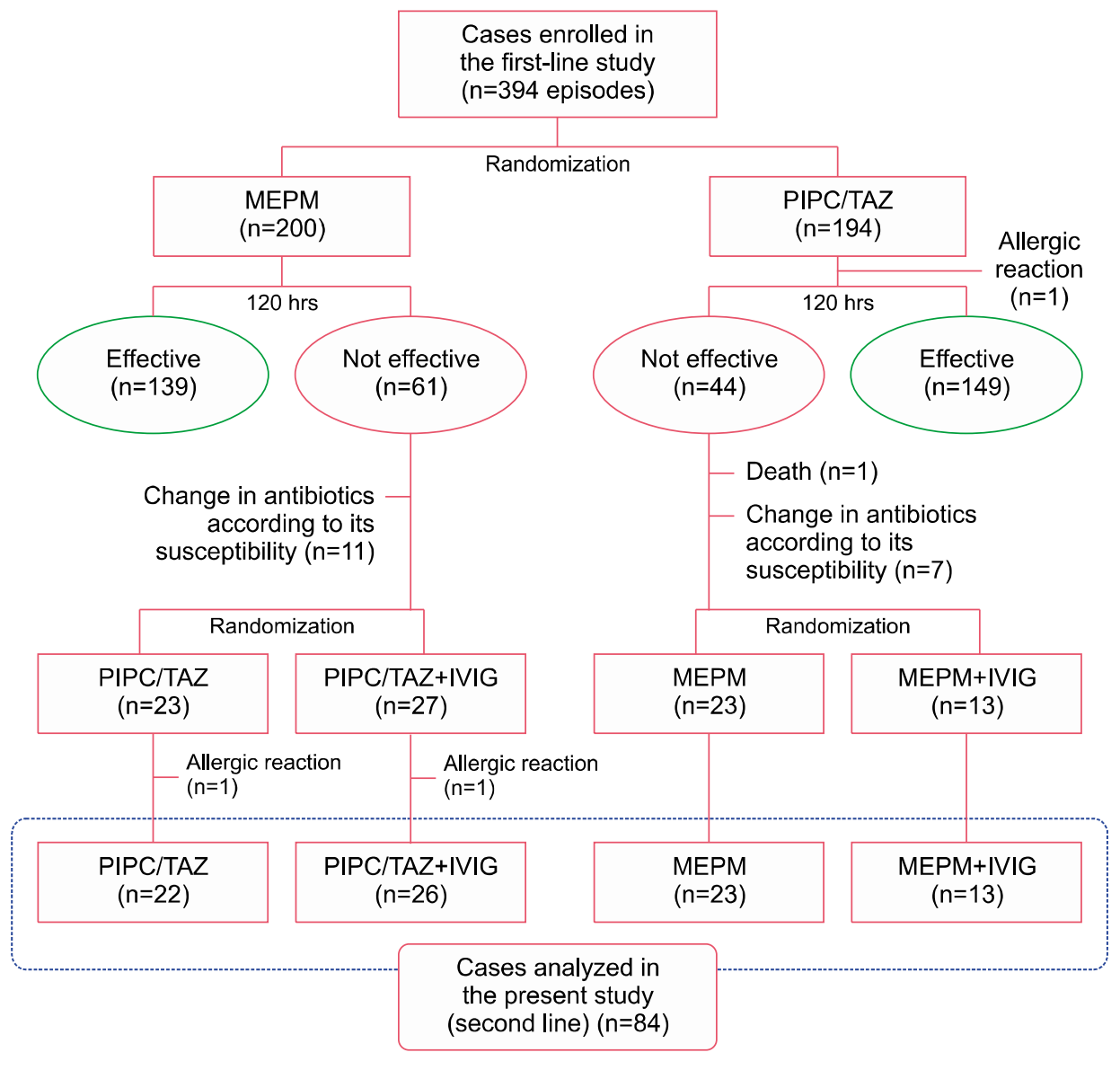

Fig. 1. Flow chart of the febrile neutropenic episodes enrolled in the present study. PIPC/TAZ, piperacillin/tazobactam; MEPM, meropenem; IVIG, intravenous immunoglobulin. 
such as coagulase-negative staphylococci, corynebacteriae other than Corynebacterium jeikeium, and other skin contaminants, were isolated, at least two consecutive blood cultures were performed to confirm that the isolates were pathogenic.

All patients enrolled in the present study were hospitalized. Prophylactic oral voriconazole at $10 \mathrm{mg} / \mathrm{kg}$ per day (maximum dose: $400 \mathrm{mg} /$ day) was prescribed for patients with acute myeloid leukemia, and oral fluconazole at $10 \mathrm{mg} / \mathrm{kg}$ per day (maximum dose: $400 \mathrm{mg} /$ day) for all other patients. Regarding HSCT patients, micafungin at $1 \mathrm{mg} / \mathrm{kg}$ per day (maximum dose: $50 \mathrm{mg} /$ day) was intravenously administered from the beginning of the preparative regimen until neutrophil recovery, followed by oral fluconazole at $10 \mathrm{mg} / \mathrm{kg}$ per day (maximum dose: $400 \mathrm{mg} /$ day) from the time of neutrophil recovery until the day of discharge.

\section{3) Statistical analysis}

Data were described using medians, ranges, and percentage values. Mann-Whitney U tests were used for comparisons of independent continuous variables, Pearson's $\chi^{2}$ tests for comparisons of categorical data, and Fisher's exact tests for categories with low numbers. Values of $P<0.05$ were considered to indicate significance. All statistical analyses were performed with EZR (Saitama Medical Center, Jichi Medical University), which is a graphical user interface for $\mathrm{R}$ (The $\mathrm{R}$ Foundation for Statistical Computing, Vienna, Austria). More precisely, it is a modified version of the $\mathrm{R}$ commander designed to add statistical functions frequently used in biostatistics [8].

\section{Results}

Eighty-six febrile episodes in 44 patients (19 females and 25 males) were enrolled in this analysis. Two patients (2 episodes) showed an allergic reaction to the drug (both were in the PIPC/TAZ group) and were excluded from the present analysis. Twenty-one patients had acute lymphoblastic leukemia, 7 acute myeloid leukemia, 6 non-Hodgkin lymphoma, 3 aplastic anemia, 3 solid tumors, 1 acute undifferentiated leukemia, and 1 each of juvenile myelomonocytic leukemia, chronic myeloid leukemia, and hemophagocytic lymphohistiocytosis. Seventeen patients underwent HSCT during the study period. Two patients had Down syndrome (2 episodes). A CV catheter was placed in 84 out of 86 episodes.

No significant differences were observed in sex, underlying disease, the treatment phase (remission induction phase after the initial diagnosis or the confirmation of relapse), prior HSCT, presence of a CV catheter, or laboratory findings (white blood cell count, C-reactive protein, $\beta$-D glucan, procalcitonin, and IgG levels) at entry between the PIPC/TAZ and MEPM groups (Table 1).

The total success rate of second-line treatment was $50.0 \%$ (42/84 episodes). In each antibiotic group, PIPC/ TAZ with or without IVIG was effective in $52.0 \%$ of the 48 episodes (53.8\% in 26 episodes treated with IVIG and $50.0 \%$ in 22 episodes treated without IVIG, $P=1.000$ ). Similarly, MEPM with or without IVIG was effective in $47.2 \%$ of the 36 episodes (53.8\% in 13 episodes treated with IVIG and $43.5 \%$ in 23 episodes treated without IVIG, $P=0.730$ ). No significant differences were observed in success rates between the PIPC/TAZ and MEPM groups ( $P=0.826$ ), irrespective of concomitant IVIG.

The efficacy of IVIG in addition to PIPC/TAZ or MEPM (IVIG+ group) in 39 episodes was analyzed and then compared with that without IVIG (IVIG - group) in 45 episodes. The characteristics of patients in the IVIG+ and IVIG - groups are shown in Table 2. No significant differences were observed in sex, underlying disease, the treatment phase (remission induction phase after the initial diagnosis or the confirmation of relapse), prior HSCT, presence of a CV catheter, or laboratory findings (white blood cell count, C-reactive protein, $\beta$-D glucan, procalcitonin, and IgG levels) at entry. No significant differences were noted in the proportions of PIPC/TAZ and MEPM between the two groups.

The success rate of the IVIG+ group was $53.8 \%$ in 39 episodes $(53.8 \%$ in 26 episodes treated with PIPC/TAZ and $53.8 \%$ in 13 episodes treated with MEPM, $P=1.000$ ), whereas that of the IVIG- group was $46.7 \%$ in 45 episodes $(50.0 \%$ in 22 episodes treated with PIPC/TAZ and 
Table 1. Demographic and clinical characteristics of patients with febrile neutropenic episodes at the beginning of second-line treatment

\begin{tabular}{|c|c|c|c|}
\hline & PIPC/TAZ $(n=48)$ & MEPM $(n=36)$ & $P$-value \\
\hline $\operatorname{Sex}(M / F)$ & $26 / 22$ & $17 / 19$ & 0.660 \\
\hline Age (median, range) & $10.9(0.5-25.4)$ & $13.55(0.4-23.7)$ & 0.658 \\
\hline Concomitant IVIG & & & 0.124 \\
\hline Yes/No & $26 / 22$ & $13 / 23$ & \\
\hline Disease & & & 0.316 \\
\hline ALL (\%) & 19 (39.6) & $18(50.0)$ & \\
\hline AML (\%) & $13(27.1)$ & $3(8.3)$ & \\
\hline NHL (\%) & $7(14.6)$ & $8(22.2)$ & \\
\hline Solid tumor $(\%)$ & $3(6.3)$ & $3(8.3)$ & \\
\hline $\mathrm{AA}(\%)$ & $3(6.3)$ & $1(2.8)$ & \\
\hline Other $(\%)$ & $3(6.3)$ & $3(8.3)$ & \\
\hline Stem cell transplantation (yes) $(\%)$ & $9(18.8)$ & $8(22.2)$ & 0.786 \\
\hline CV catheter (yes) (\%) & $47(97.9)$ & $35(97.2)$ & 1.000 \\
\hline $\begin{array}{l}\text { WBC at the beginning of treatment } \\
\left(\times 10^{9} / \mathrm{L} \text {, median, range }\right)\end{array}$ & $0.28(0.01-2.00)$ & $0.26(0.01-14.00)$ & 0.553 \\
\hline $\mathrm{CRP}$ at entry (mg/dL, median, range) & $3.84(0.05-29.96)$ & $3.93(0.05-15.98)$ & 0.845 \\
\hline$\beta$-D glucan (pg/mL, range) & $6.9(1.6-53.2)$ & $8.9(1.9-20.9)$ & 0.172 \\
\hline Procalcitonin $(\mathrm{ng} / \mathrm{mL}$, range) & $0.23(0.03-51.03)$ & $0.25(0.04-46.63)$ & 0.863 \\
\hline $\operatorname{lgG}(\mathrm{mg} / \mathrm{dL}$, range $)$ & $673(247-1502)$ & $673(59-1255)$ & 0.970 \\
\hline
\end{tabular}

M, male; F, female; IVIG, intravenous immunoglobulin; ALL, acute lymphoblastic leukemia; AML, acute myeloid leukemia; NHL, non-Hodgkin lymphoma; AA, aplastic anemia; CV, central venous; WBC, white blood cell count; CRP, C-reactive protein; IgG, immunoglobulin G.

Table 2. Demographic and clinical characteristics of patients with febrile neutropenic episodes in IVIG + and IVIG - groups at the beginning of second-line treatment

\begin{tabular}{|c|c|c|c|}
\hline & $\mathrm{IVIG}+(\mathrm{n}=39)$ & $I V I G-(n=45)$ & $P$-value \\
\hline $\operatorname{Sex}(M / F)$ & $24 / 15$ & $19 / 26$ & 0.086 \\
\hline Age (median, range) & $11.5(0.4-25.4)$ & $11.8(0.5-24.3)$ & 0.796 \\
\hline Antibiotics & & & 0.124 \\
\hline PIPC/TAZ (\%) & $26(66.7)$ & $22(48.9)$ & \\
\hline MEPM (\%) & $13(33.3)$ & $23(51.1)$ & \\
\hline Disease & & & 0.078 \\
\hline ALL (\%) & $18(46.2)$ & $19(42.2)$ & \\
\hline AML $(\%)$ & $11(28.2)$ & $5(11.1)$ & \\
\hline NHL $(\%)$ & $6(15.4)$ & $9(20.0)$ & \\
\hline Solid tumor $(\%)$ & $3(7.7)$ & $3(6.7)$ & \\
\hline $\mathrm{AA}(\%)$ & $1(2.6)$ & $3(6.7)$ & \\
\hline Other $(\%)$ & $0(0)$ & $6(13.3)$ & \\
\hline Stem cell transplantation (yes) (\%) & $6(15.4)$ & $11(24.4)$ & 0.416 \\
\hline CV catheter (yes) (\%) & $38(97.4)$ & $44(97.8)$ & 1.000 \\
\hline $\begin{array}{l}\text { WBC at the beginning of treatment } \\
\left(\times 10^{9} / \mathrm{L} \text {, median, range }\right)\end{array}$ & $0.37(0.01-14.00)$ & $0.27(0.01-4.92)$ & 0.589 \\
\hline $\mathrm{CRP}$ at entry (mg/dL, median, range) & $4.93(0.05-29.96)$ & $3.78(0.05-28.49)$ & 0.615 \\
\hline$\beta$-D glucan (pg/mL, range) & $7.9(1.6-53.2)$ & $7.9(1.6-20.9)$ & 0.842 \\
\hline Procalcitonin (ng/mL, range) & $0.24(0.03-51.03)$ & $0.22(0.04-46.63)$ & 0.935 \\
\hline $\operatorname{lgG}(\mathrm{mg} / \mathrm{dL}$, range $)$ & $682(59-1255)$ & 664 (110-1502) & 0.496 \\
\hline
\end{tabular}

M, male; F, female; PIPC/TAZ, piperacillin tazobactam; MEPM, meropenem; ALL, acute lymphoblastic leukemia; AML, acute myeloid leukemia; NHL, non-Hodgkin lymphoma; AA, aplastic anemia; CV, central venous; WBC, white blood cell count; CRP, C-reactive protein; IgG, immunoglobulin G. 
43.5\% in 23 episodes treated with MEPM, $P=0.768$ ). No significant differences were observed in success rates between episodes treated with and without IVIG $(P=0.662)$. Success rates in episodes with serum IgG lower than $500 \mathrm{mg} / \mathrm{dL}$ at the beginning of the antibiotic treatment were $50 \%$ in both the IVIG+ and IVIG - groups ( $P=1.000)$, whereas those in episodes with serum IgG higher than $500 \mathrm{mg} / \mathrm{dL}$ were $54.8 \%$ in the IVIG+ group and $45.7 \%$ in the IVIG - group ( $P=0.622)$.

An analysis of the ages of effective and ineffective cases in the IVIG+ group revealed that effective cases $(n=21)$ were slightly younger than ineffective cases $(\mathrm{n}=18)$ [median age: 6.7 (range, 0.5-25.0) vs 13.7 (range, 0.4-25.4) years, $P=0.143]$. The age with the highest sensitivity and specificity was estimated to be 8.0 years in a receiver operating characteristic (ROC) curve analysis (sensitivity and specificity were 61.9 and $78.9 \%$, respectively). In the IVIG+ group, the success rate in patients younger than 8 years was $78.6 \%(11 / 14)$, which was significantly higher than that in patients aged 8 years and older (40.0\% (10/25), $P=0.043)$. On the other hand, in the IVIG - group, no significant differences were observed in success rates between patients younger than or aged 8 years and older (42.9\% vs $48.4 \%$, respectively, $P=0.759$ ) (Fig. 2). These results indicate that concomitant IVIG was significantly

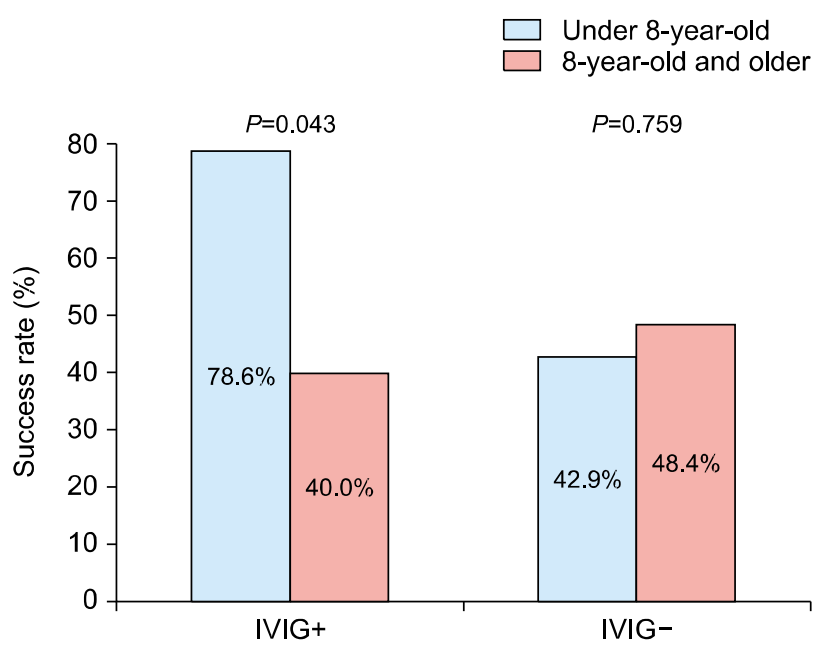

Fig. 2. Comparison of success rates between second-line treatment with $(\mathrm{IVIG}+)$ and without concomitant IVIG (IVIG-) depicted as a function of age (younger than 8 years vs. 8 years and older). IVIG, intravenous immunoglobulin. more effective in patients younger than 8 years than in those aged 8 years and older.

Table 3 summarizes the comparison of success rates among items analyzed in the present study.

Regarding adverse events, one patient treated with $\mathrm{PIPC/TAZ} \mathrm{as} \mathrm{second-line} \mathrm{treatment} \mathrm{developed} \mathrm{liver} \mathrm{dys-}$ function [grade 4 elevation in aminotransferase by the Common Terminology Criteria for Adverse Events v4.0 (CTCAE)], which was attenuated by the discontinuation of PIPC/TAZ. No other obvious adverse events associated with the antibiotic treatment or IVIG were observed.

\section{Discussion}

The management of $\mathrm{FN}$ is important in the management of pediatric hematological and malignant diseases. The efficacy of first-line antibiotic treatment has been investigated; however, limited information is currently available on salvage therapy for cases refractory to this treatment. We previously conducted two prospective studies on second-line antibiotic treatment. In the first study, we demonstrated that MEPM was effective and safe as second-line treatment for cases refractory to first-line treatment (cefepime or PIPC/TAZ). The findings obtained also indicated that the combination of IVIG in addition to MEPM was slightly more effective in cases with low serum IgG levels $(<500 \mathrm{mg} / \mathrm{dL})$ than in those with IgG higher than $500 \mathrm{mg} / \mathrm{dL}$ [3]. In the second study, PIPC/TAZ was found to be as effective and safe as MEPM when it was used in second-line treatment for cases refractory to first-line MEPM (approximately 50\% of cases with MEPM treatment failure were rescued by PIPC/TAZ and vice versa). In that study, cases treated with concomitant IVIG had slightly higher success rates than those without IVIG (58.7\% vs. $41.9 \%, P=0.064)$. Moreover, when cases were restricted to those with $\operatorname{IgG}<500 \mathrm{mg} / \mathrm{dL}$ at the beginning of second-line treatment, success rates were significantly higher in cases with than in those without IVIG ( $81.8 \%$ vs. $38.5 \%, P=0.047)$ [4]. The comparison of MEPM and PIPC/TAZ performed in the present study was the same as that in the previous study [4]; however, the dose and administration of PIPC/TAZ were 
Table 3. Comparison of success rates (\%) summarized

\begin{tabular}{|c|c|c|c|c|c|c|}
\hline & Total $\mathrm{N}$ & Success N (\%) & & Total N & Success N (\%) & $P$-value \\
\hline \multicolumn{7}{|c|}{ In patients treated with PIPC/TAZ } \\
\hline Treated with IVIG & 26 & $14(53.8)$ & Treated without IVIG & 22 & $11(50.0)$ & 1.000 \\
\hline \multicolumn{7}{|c|}{ In patients treated with MEPM } \\
\hline Treated with IVIG & 13 & $7(53.8)$ & Treated without IVIG & 23 & $10(43.5)$ & 0.730 \\
\hline \multicolumn{7}{|l|}{ In patients treated with IVIG } \\
\hline Treated with PIPC/TAZ & 26 & $14(53.8)$ & Treated with MEPM & 13 & $7(53.8)$ & 1.000 \\
\hline \multicolumn{7}{|c|}{ In patients treated without IVIG } \\
\hline Treated with PIPC/TAZ & 22 & $11(50.0)$ & Treated with MEPM & 23 & $10(43.5)$ & 0.768 \\
\hline \multicolumn{7}{|l|}{ In all patients } \\
\hline Treated with IVIG & 39 & $21(53.8)$ & Treated without IVIG & 45 & $21(46.7)$ & 0.662 \\
\hline \multicolumn{7}{|c|}{ In patients with serum IgG lower than $500 \mathrm{mg} / \mathrm{dL}$} \\
\hline Treated with IVIG & 8 & $4(50.0)$ & Treated without IVIG & 10 & $5(50.0)$ & 1.000 \\
\hline \multicolumn{7}{|c|}{ In patients with serum IgG higher than $500 \mathrm{mg} / \mathrm{dL}$} \\
\hline Treated with IVIG & 31 & $17(54.8)$ & Treated without IVIG & 35 & $16(45.7)$ & 0.622 \\
\hline \multicolumn{7}{|c|}{ In patients younger than 8 years } \\
\hline Treated with IVIG & 14 & $11(78.6)$ & Treated without IVIG & 14 & $6(42.9)$ & 0.120 \\
\hline \multicolumn{7}{|c|}{ In patients aged 8 years and older } \\
\hline Treated with IVIG & 25 & $10(40.0)$ & Treated without IVIG & 31 & $15(48.4)$ & 0.596 \\
\hline \multicolumn{7}{|l|}{ In patients treated with IVIG } \\
\hline Younger than 8 years & 14 & $11(78.6)$ & Aged 8 years and older & 25 & $10(40.0)$ & 0.043 \\
\hline \multicolumn{7}{|c|}{ In patients treated without IVIG } \\
\hline Younger than 8 years & 14 & $6(42.9)$ & Aged 8 years and older & 31 & $15(48.4)$ & 0.759 \\
\hline
\end{tabular}

PIPC/TAZ, piperacillin tazobactam; IVIG, intravenous immunoglobulin; MEPM, meropenem; IgG, immunoglobulin G.

changed from the previous study [5]. The administration procedure, which was $337.5 \mathrm{mg} / \mathrm{kg} /$ day three times a day in the previous study, was changed to $360 \mathrm{mg} / \mathrm{kg} / \mathrm{day}$ four times a day. These changes in the dose and administration procedure were expected to increase the time above the minimal inhibitory concentration (MIC), which was related to the efficacy of $\beta$-lactam antibiotics, including PIPC/TAZ, resulting in better efficacy in first-line treatment than that in the previous study [9]; the success rate of PIPC/TAZ increased from 62.4\% (previous study) to $77.2 \%$ (present study) ( $P=0.001$ ). The total efficacy of first-line treatment in the present study was $73.3 \%$ (77.2\% in the PIPC/TAZ group and 69.5\% in the MEPM group), which was higher than that in the previous study of 64.1\% (62.4\% in the PIPC/TAZ group and 65.9\% in the MEPM group) ( $P=0.004)$. However, the total efficacy of second-line treatment in the present study was $50.0 \%$, which was as effective as that in the previous study (49.3\%) [4]. Success rates did not significantly differ between cases treated with PIPC/TAZ (switched from MEPM with or without IVIG) and with MEPM (switched from PIPC/TAZ with or without IVIG) (52.0\% vs. $47.2 \%, P=0.826)$. Although PIPC/TAZ is considered to have a narrower spectrum than MEPM, approximately $50 \%$ of the cases that did not respond to first-line MEPM were rescued by second-line PIPC/TAZ. One of the pathogens known to be resistant to MEPM, but susceptible to PIPC/TAZ is Enterococcus spp. This bacterium was not detected in blood cultures from patients undergoing first- and second-line treatments in the present study; however, we cannot exclude the possibility that Enterococcus spp. may have been associated with $\mathrm{FN}$ in several patients.

Although the dose and administration procedure of PIPC/TAZ have been changed from the previous study [4], the frequency of grade 2 or higher liver dysfunction according to CTCAE v4.0 in patients treated with PIPC/ TAZ in the present study was $2.1 \%$, which was not statistically different from the previous study (6.2\%) [4]. The dose of MEPM in the present study was relatively higher than that used in general pediatric practice $(60 \mathrm{mg} / \mathrm{kg} /$ 
day); however, no obvious adverse events associated with MEPM was observed in this study. Therefore, we believe that the doses of PIPC/TAZ and MEPM used in the present study were considered to be safe and tolerable.

The efficacy of IVIG was also evaluated in the present study. The combination of IVIG plus antibiotics has been reported as an effective treatment for severe infectious diseases [10-13]. IVIG contains antibodies that are specific for a number of bacteria or bacterial toxins. Although the mechanisms underlying antibacterial activity mediated by IVIG involve bacteriolysis, the neutralization of toxins, and opsonization, its ability to increase bacterial susceptibility to antibiotics remains unclear. Nevertheless, concomitant IVIG is considered to be an important therapeutic option because its antibacterial activity is independent of the mechanisms underlying drug resistance [14]. With this background, IVIG may be effective against drug-resistant bacteria, such as methicillinresistant Staphylococcus aureus (MRSA) [15,16].

We previously reported that the concomitant use of IVIG was effective in patients with low serum IgG levels at the start of second-line antibiotics [4]. However, the present study failed to demonstrate this effectiveness in cases irrespective of their serum IgG levels. One possible explanation for this discrepancy may be that the increased efficacy of first-line treatment may have made it difficult to statistically prove the efficacy of IVIG over that in the previous study, particularly in cases with first-line PIPC/TAZ [4].

A novel result regarding IVIG in the present study was the significantly better efficacy of concomitant IVIG in patients younger than 8 years than in those aged 8 years and older. To the best of our knowledge, it has not yet been established whether the efficacy of concomitant IVIG against infectious diseases varies with age. Moreover, regarding the effects of IVIG on other diseases, such as autoimmune diseases, it currently remains unclear whether differences in efficacy are dependent on age. It is difficult to explain why concomitant IVIG was less effective in patients older than 8 years. Although not closely related with FN, we previously reported that the risk of developing IFI was high in cases older than 9 years
[17]. The similarity in patient ages between those at risk of developing IFI (older than 9 years) and those with decreased IVIG effectiveness (older than 8 years) indicate that both phenomena are associated with the development of immune function, such as lymphoid tissue [18]. Further studies are needed on the relationship between IVIG efficacy and age.

The optimal dose of IVIG used together with antibiotics in pediatric patients with FN has not been determined. We set the dose of IVIG in the present study based on the dose approved for sever infections in Japan. There have been several studies on the administration of IVIG for severe infections. As a result of the multicenter randomized controlled trial (RCT) in adult patients with sepsis, no improvement in survival rate was observed in the group receiving a total of $0.9 \mathrm{~g} / \mathrm{kg}(0.6 \mathrm{~g} / \mathrm{kg}$ on day $1,0.3 \mathrm{~g} / \mathrm{kg}$ on day 2) of IVIG [19]. Besides, as a result of the multicenter RCT for neonates with sepsis, no improvements in survival rate or incidence of sequelae were observed in the group receiving IVIG $500 \mathrm{mg} / \mathrm{kg}$ twice (48 hour-interval) [20]. On the other hand, regarding reports that IVIG was effective, RCT in infants with severe infection aged 1 to 24 months admitted to pediatric intensive care unit showed improvement in survival and length of stay in the group receiving IVIG $400 \mathrm{mg} / \mathrm{kg}$ for 3 days [21]. However, since the last report was the result of a small-scale RCT in a single institution, largescale multicenter RCTs or meta-analysis for pediatric patients are warranted in the future.

In conclusion, the present study demonstrated that PIPC/TAZ and MEPM were equally effective and safe as second-line treatments for FN. Approximately $50 \%$ of cases that did not respond to first-line MEPM were rescued by second-line PIPC/TAZ and vice versa. In addition, concomitant IVIG was effective, particularly in patients younger than 8 years.

\section{Acknowledgments}

No funding was secured for study.

The authors would like to thank Ms. Yukiko Shiota for data management. 


\section{Conflict of Interest Statement}

The authors have no conflict of interest to declare.

\section{References}

1. Hann I, Viscoli C, Paesmans M, Gaya H, Glauser M. A comparison of outcome from febrile neutropenic episodes in children compared with adults: results from four EORTC studies. International Antimicrobial Therapy Cooperative Group (IATCG) of the European Organization for Research and Treatment of Cancer (EORTC). Br J Haematol 1997;99: 580-8.

2. Viscoli C, Moroni C, Boni L, et al. Ceftazidime plus amikacin versus ceftazidime plus vancomycin as empiric therapy in febrile neutropenic children with cancer. Rev Infect Dis 1991; 13:397-404.

3. Kobayashi R, Suzuki D, Sano H, Kishimoto K, Yasuda K, Kobayashi K. Effect of meropenem with or without immunoglobulin as second-line therapy for pediatric febrile neutropenia. Pediatr Int 2014;56:526-9.

4. Kobayashi R, Hori D, Sano H, Suzuki D, Kishimoto K, Kobayashi K. Meropenem versus piperacillin/tazobactam with or without immunoglobulin as second-line therapy for febrile neutropenia in pediatric patients. J Microbiol Immunol Infect 2018;51:473-7.

5. Kobayashi R, Sano H, Matsushima S, et al. Meropenem versus piperacillin/tazobactam for febrile neutropenia in pediatric patients: efficacy of piperacillin/tazobactam as a 1-h drip infusion four times a day. Int J Hematol 2021;113:430-5.

6. Schulz KF, Altman DG, Moher D; CONSORT Group. CONSORT 2010 statement: updated guidelines for reporting parallel group randomised trials. BMJ 2010;340:c332.

7. Freifeld AG, Bow EJ, Sepkowitz KA, et al. Clinical practice guideline for the use of antimicrobial agents in neutropenic patients with cancer: 2010 update by the Infectious Diseases Society of America. Clin Infect Dis 2011:52:427-31.

8. Kanda Y. Investigation of the freely available easy-to-use software 'EZR' for medical statistics. Bone Marrow Transplant 2013;48:452-8.

9. Sano H, Kobayashi R, Suzuki D, Hori D, Kishimoto K, Kobayashi K. A prospective randomized trial comparing piperacillin/tazobactam with meropenem as empirical antibiotic treatment of febrile neutropenic children and adolescents with hematologic and malignant disorders. Pediatr
Blood Cancer 2017;64.

10. Masaoka T, Hasegawa H, Takaku F, et al. The efficacy of intravenous immunoglobulin in combination therapy with antibiotics for severe infections. Jap J Chemother 2000;48:199217.

11. Schedel I, Dreikhausen U, Nentwig B, et al. Treatment of gram-negative septic shock with an immunoglobulin preparation: a prospective, randomized clinical trial. Crit Care Med 1991;19:1104-13.

12. Werdan K, Pilz G. Supplemental immune globulins in sepsis: a critical appraisal. Clin Exp Immunol 1996;104 Suppl 1: 83-90.

13. Laupland KB, Kirkpatrick AW, Delaney A. Polyclonal intravenous immunoglobulin for the treatment of severe sepsis and septic shock in critically ill adults: a systematic review and meta-analysis. Crit Care Med 2007;35:2686-92.

14. Itoh H, Matsuo H, Kitamura N, et al. Enhancement of neutrophil autophagy by an IVIG preparation against multidrug-resistant bacteria as well as drug-sensitive strains. J Leukoc Biol 2015;98:107-17.

15. Farag N, Mahran L, Abou-Aisha K, El-Azizi M. Assessment of the efficacy of polyclonal intravenous immunoglobulin $G$ (IVIG) against the infectivity of clinical isolates of methicillin-resistant Staphylococcus aureus (MRSA) in vitro and in vivo. Eur J Clin Microbiol Infect Dis 2013;32:1149-60.

16. Matsuo $\mathrm{H}$, Itoh $\mathrm{H}$, Kitamura $\mathrm{N}$, et al. Intravenous immunoglobulin enhances the killing activity and autophagy of neutrophils isolated from immunocompromised patients against multidrug-resistant bacteria. Biochem Biophys Res Commun 2015;464:94-9.

17. Kobayashi R, Hori D, Sano H, Suzuki D, Kishimoto K, Kobayashi K. Risk factors for invasive fungal infection in children and adolescents with hematologic and malignant diseases: a 10-year analysis in a single institute in Japan. Pediatr Infect Dis J 2018;37:1282-5.

18. Scammon, RE. The measurement of the body in childhood. In: Harris JA, Jackson CM, Paterson DG, Scammon RE, editors. The measurement of man. Minneapolis: University of Minnesota Press, 1930;173-215.

19. Werdan K, Pilz G, Bujdoso O, et al. Score-based immunoglobulin G therapy of patients with sepsis: the SBITS study. Crit Care Med 2007:35:2693-701.

20. Brocklehurst P, Farrell B, King A, et al. Treatment of neonatal sepsis with intravenous immune globulin. N Engl J Med 2011; 365:1201-11.

21. El-Nawawy A, El-Kinany H, Hamdy El-Sayed M, Boshra N. Intravenous polyclonal immunoglobulin administration to sepsis syndrome patients: a prospective study in a pediatric intensive care unit. J Trop Pediatr 2005;51:271-8. 